OPEN ACCESS

Edited by:

Luis A. Martinez-Lemus,

University of Missouri, USA

Reviewed by:

Zhe Sun,

University of Missouri, USA

Zhongkui Hong,

University of South Dakota, USA

*Correspondence:

Mika P. Tarvainen

mika.tarvainen@uef.fi

Specialty section:

This article was submitted to

Vascular Physiology,

a section of the journal

Frontiers in Physiology

Received: 01 November 2016 Accepted: 12 December 2016

Published: 27 December 2016

Citation:

Yli-Ollila H, Tarvainen MP, Laitinen TP and Laitinen TM (2016) Transfer Function Analysis of the Longitudinal Motion of the Common Carotid Artery

Wall. Front. Physiol. 7:651

doi: 10.3389/fphys.2016.00651

\section{Transfer Function Analysis of the Longitudinal Motion of the Common Carotid Artery Wall}

\author{
Heikki Yli-Ollila ${ }^{1,2,3}$, Mika P. Tarvainen ${ }^{1,2 *}$, Tomi P. Laitinen ${ }^{1,4}$ and Tiina M. Laitinen ${ }^{1}$ \\ ${ }^{1}$ Department of Clinical Physiology and Nuclear Medicine, Kuopio University Hospital (KYS), Kuopio, Finland, ${ }^{2}$ Department of \\ Applied Physics, University of Eastern Finland (UEF), Kuopio, Finland, ${ }^{3}$ Department of Radiology, Kanta-Häme Central \\ Hospital, Hämeenlinna, Finland, ${ }^{4}$ Department of Clinical Physiology and Nuclear Medicine, University of Eastern Finland \\ (UEF), Kuopio, Finland
}

The longitudinal motion of the carotid wall is a potential new measure of arterial stiffness. Despite the over decade long research on the subject, the driving force and the specific longitudinal kinetics of the carotid wall has remained unclear. In this study, a transfer function analysis with 20 healthy subjects is presented to derive how the energy from the blood pressure moves the innermost arterial wall longitudinally and how the kinetic energy is then transferred to the outermost arterial layer. The power spectrums display that the main kinetic energy of the longitudinal motion is on band $\mathrm{O}-3 \mathrm{~Hz}$ with a peak on the $1.1 \mathrm{~Hz}$ frequency. There is a large variation among the individuals, how the energy from the blood pressure transfers into the longitudinal motion of the arterial wall since the main direction of the longitudinal motion varies individually and because early arterial stiffening potentially has an effect on the time characteristics of the energy transfer. The energy transfer from the innermost to the outermost wall layer is more straightforward: on average, a $17 \%$ of the longitudinal amplitude is lost and an $18.9 \mathrm{~ms}$ delay is visible on the $1.0 \mathrm{~Hz}$ frequency.

Keywords: adventitia, arterial stiffness, blood pressure, frequency space, intima-media, motion tracking, power spectrum, ultrasound imaging

\section{INTRODUCTION}

Longitudinal motion of the carotid artery wall is an interesting phenomenon with partly unknown origin. Ultrasound imaging has proven to be a good technique to measure the phenomenon (Golemati et al., 2003; Persson et al., 2003; Svedlund and Gan, 2011a; Zahnd et al., 2011b; YliOllila et al., 2013; Albinsson et al., 2014). Most of the longitudinal shear is believed to be between the media and the adventitia layers of the arterial wall (Nilsson et al., 2010). Due to the low contrast of the media layer in the ultrasound images, intima and media layers are usually measured as a one complex (Cinthio et al., 2006). The amplitude of the longitudinal motion of the carotid wall has been shown to be liked to well-known stiffness indices: a direct correlation to carotid artery distensibility and an inverse correlation to pulse wave velocity has been found (Taivainen et al., 2015). In addition, the reduction of the amplitude of the longitudinal motion of the carotid intima layer has been linked to aging, hypertension, diabetes, plaque burden in the common carotid artery and the risk of myocardial ischemia and cardiovascular disease (Svedlund and Gan, 2011b; Svedlund et al., 2011; Zahnd et al., 2011a, 2012; Tat et al., 2015). In animal models, the reduction of the amplitude of the longitudinal carotid wall motion has been associated with plaque burden 
in brachiocephalic artery as well as with the total cholesterol level in blood (Svedlund and Gan, 2011b). However, recent results display that focusing on the amplitude of the longitudinal motion may be a restrictive approach, the fine structure of the motion has also potential to reveal the status of the vascular system (Taivainen et al., 2015; Yli-Ollila et al., 2015, 2016).

Transfer function analysis is a widely used tool in electronics to reveal linear relationship between two connected signals. In the field of cardiovascular research, the transfer function analysis has been used in studies of vascular function to model aortic pressure curve by measuring the pressure only from superficial arteries (Karamanoglu and Feneley, 1996; Segers et al., 2000). In addition, the transfer function analysis has been used to investigate the relationship between arterial blood pressure and cerebral blood flow (Kuo et al., 1998), to identify high-grade carotid stenosis with impaired cerebral autoregulation (Hu et al., 1999) and to study the effect of aging and hypertension on cerebral artery blood flow (Lipsitz et al., 2000). Nevertheless, the transfer function analysis has not previously been used to investigate the longitudinal motion of the artery wall.

The aim of this study is to use the transfer function analysis to characterize the longitudinal motion between the intimamedia complex and the adventitia layer as well as blood pressure and intima-media complex in healthy subjects in detail. Using referential stiffness measurements, the spectral characteristics of the transfer function of the longitudinal motion are investigated as potential indicators of early arterial stiffening.

\section{MATERIALS AND METHODS}

\section{Subjects and Study Protocol}

Twenty healthy, non-smoking volunteers with no history of cardiac diseases participated in the study. Ten of the volunteers were men and 10 were women. One male subject was omitted from the analysis because during measurement a left bundle branch block was found and the subject could not be presented as a healthy volunteer. The clinical characteristics of the study population have been presented in Table 1. First, the volunteer was positioned in the supine position and ECG-electrodes were attached on the chest. Then the volunteer was allowed to rest for $10 \mathrm{~min}$ and the blood pressure was measured from the left upper arm using an automatic blood pressure monitor (Omron, M4-I, Matsusaka, Japan). Immediately after the blood pressure measurement, an anatomical B-mode ultrasound video was imaged from the left common carotid artery using $18 \mathrm{MHz}$ linear transducer (Philips EPIQ 7, L18-5 transducer, Best, The Netherlands). The ultrasound transducer was handled by an experienced physician and positioned on the carotid artery, thus the bifurcation was merely visible on the right side of the ultrasound image. The size of the ultrasound frame was $1.25 \times$ $1.5 \mathrm{~cm}$ (width $\times$ height) and the used imaging frame rate was $85 \mathrm{~Hz}$. A total of 5-min acquisition was achieved by collecting a set of thirteen $10 \mathrm{~s}$ ultrasound videos. After the ultrasound acquisition, the blood pressure was measured again and an average of the two blood pressure measurements was calculated.

Finally an applanation tonometry measurement was performed on the subject using SphygmoCor (version 9, AtCor
Medical Inc., Itasca, IL, USA). In the applanation tonometry measurement, a pen like pressure probe (SPT-301B, Millar instruments, Houston, TX, USA) was positioned on the radial artery of the subject. The SphygmoCor system measures the average pressure waveform of the radial artery and by using in-build transfer function analysis it computes the pressure waveform in the aorta and in the carotid artery. A widely used arterial stiffness measure augmentation index adjusted for the heart rate of 75 beats per minute (Aix@75) was obtained from the system and SphygmoCor system was also used to transform the systolic and diastolic upper arm pressures (averages of the two measurements) into carotid pressures.

All volunteers were prohibited from drinking coffee for $2 \mathrm{~h}$ before the experiment. Fully informed written consent was obtained from each participant and the Ethics Committee of the University of Eastern Finland and Kuopio University Hospital approved the used study protocol.

\section{Motion Tracking and Blood Pressure Curve Calculation}

The longitudinal motion tracking from the B-mode videos was performed in Matlab (R2013b, The MathWorks Inc., Natick, MA, USA) using our in-house-build motion tracking program, including contrast optimization (Yli-Ollila et al., 2013). One region of interest (ROI) was positioned on the intima-media complex of the far carotid wall and another ROI was positioned on the adventitia layer and third referential ROI on surrounding tissues behind the carotid artery, see Figure 1. To simultaneously track the diameter change of the artery for blood pressure curve calculations, additional ROIs were positioned on the near and far carotid wall. The sizes of the ROIs were otherwise freely selectable but the widths of the ROIs were forced to be the same. The average size of the intima-media, adventitia and surrounding tissue ROIs were (width $\times$ height) $2.58 \times 0.33 \mathrm{~mm}, 2.58 \times$ $0.30 \mathrm{~mm}$, and $2.58 \times 1.15 \mathrm{~mm}$, respectively. The average sizes of the ROIs used for the diameter tracking were $2.58 \times 0.97 \mathrm{~mm}$ for the near wall and $2.58 \times 1.83 \mathrm{~mm}$ for the far wall.

To ensure accurate measurement, the motion tracking was done in heartbeat long sequences and the accuracy of the motion tracking was monitored by an experienced physicist after every cardiac cycle. If there were problems (i.e., swallowing) in the longitudinal motion traces, the observed heartbeat long signal was cut out from the transfer function analysis. The motion of the surrounding tissue was eliminated from the final longitudinal intima-media and adventitia traces, i.e., the recorded longitudinal motion of the surrounding tissue ROI was subtracted from the recorded longitudinal motion of the intima-media and adventitia ROIs.

The carotid blood pressure signal was computed from the carotid diameter change curve. The values of the diameter curve were linearly changed to be the blood pressure values subject wise by computing the average diameter curve and setting the systolic and diastolic diameter to average systolic and diastolic blood pressure, obtained from the blood pressure measurements before and after the ultrasound scanning. The linear relationship between blood pressure and artery diameter has been found 
TABLE 1 | Clinical characteristics of the study population.

\begin{tabular}{|c|c|c|c|c|c|c|}
\hline & Age (years) & Height (cm) & Weight (kg) & Brachial SBP/DBP $(\mathrm{mmHg})$ & Carotid SBP/DBP (mmHg) & Heart rate $(\mathrm{Hz})$ [bpm] \\
\hline Avg & 29 & 174 & 69 & $115 / 68$ & $121 / 69$ & 1.09 [65] \\
\hline$S D$ & 7 & 10 & 10 & $11 / 6$ & $13 / 6$ & $0.16[10]$ \\
\hline Range & $19-49$ & $155-189$ & $52-85$ & $96-135 / 60-84$ & $96-141 / 60-84$ & $0.75-1.36$ [45-82] \\
\hline
\end{tabular}

Avg, average; SD, standard deviation; SBP, systolic blood pressure; DBP, diastolic blood pressure. $n=19$ (9 men, 10 women).



FIGURE 1 | Longitudinal view of the left common carotid artery, imaged $5 \mathrm{~mm}$ to caudal direction from the carotid bifurcation. The typical locations of the regions of interest $(\mathrm{ROI})$ used in the tracking of the longitudinal motion of the carotid wall are presented with white color boxes. $\mathrm{ROI}$ of the intima-media complex, solid line; $\mathrm{ROI}$ of the adventitia layer, dashed line; $\mathrm{ROI}$ of the surrounding tissue, dotted line.

previously (Barnett et al., 1961; Patel et al., 1963; Sugawara et al., 2000; Giannattasio et al., 2008).

\section{Longitudinal Motion Parameters}

From the 5-min longitudinal motion tracings, an average, heartbeat long longitudinal motion signal was computed for every subject. From this average motion curve, four longitudinal motion parameters were computed: $\mathrm{IO}_{\mathrm{ampl}}$, peak-to-peak amplitude of the longitudinal motion of intima-media; $\mathrm{IO}_{\text {ante }}$, antegrade (in the direction of the main blood flow) component of the longitudinal amplitude; $\mathrm{IO}_{\text {retro, }}$ retrograde (against the direction of the main blood flow) component of the longitudinal amplitude; $\mathrm{IO}_{\mathrm{dev}}$, average deviation of the longitudinal trace from the baseline during a heartbeat. The baseline is the longitudinal

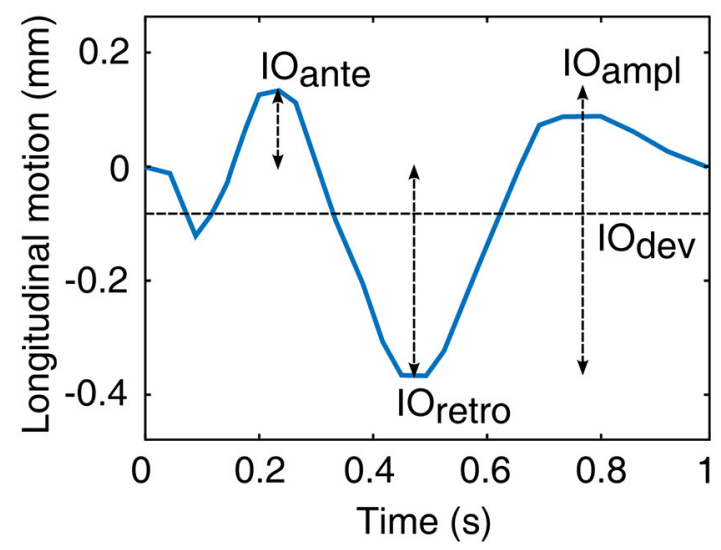

FIGURE 2 | Illustration of the motion parameters derived from the longitudinal motion curve between the intima-media complex of the common carotid artery wall and surrounding tissues (origo). $1 \mathrm{O}_{\mathrm{ampl}}$, peak-to-peak amplitude; $1 \mathrm{O}_{\text {ante }}$, antegrade component of the peak-to-peak amplitude; $1 O_{\text {retro }}$, retrograde component of the peak-to-peak amplitude;

$1 \mathrm{O}_{\mathrm{dev}}$, average deviation from the baseline.

position of the intima-media at the moment of ECG R-spike. $\mathrm{IO}_{\mathrm{dev}}$ describes the main direction of the longitudinal motion: minus sign represents motion into the retrograde direction and plus sign represents motion into the antegrade direction. See Figure 2 for illustration of the longitudinal motion parameters.

\section{Transfer Function Analysis}

Two different transfer functions were computed in this study: one between the longitudinal motion of the intima-media complex (input) and the adventitia layer (output) and another between the carotid blood pressure (input) and the longitudinal motion of the intima-media complex (output). In addition to the average transfer functions of the whole population, the same transfer functions were computed separately for the upper and lower quartiles of the data arranged according to the average deviation of the longitudinal motion waveform of the intimamedia complex $\left(\mathrm{IO}_{\mathrm{dev}}\right)$. Multiple subject specific waveforms have been observed in the longitudinal motion of the common carotid artery (Cinthio et al., 2006; Ahlgren et al., 2012; Yli-Ollila et al., 2013, 2016), thus the impact of the different waveforms on the transfer functions are observed here.

The transfer function analysis was performed separately for every continuous, good quality part of the separate 10-s videos. The length of these segments varied from one successful, heartbeat long motion tracking to $10 \mathrm{~s}$ (length of the entire 
video). First, the trend in the signals was removed using linear detrending and then the signals were windowed using a periodic Hanning window to avoid spectral leakage. Next, the signals, which all had the sampling frequency of $85 \mathrm{~Hz}$, were zero-padded to the same length of 1000 samples to simplify the calculations. The power spectral densities of the input and output signals as well as the cross power spectrum were computed as follows:

$$
\begin{aligned}
& P_{x x}(f)=\frac{1}{L F_{s} U} X(f) X^{*}(f), \\
& P_{y y}(f)=\frac{1}{L F_{s} U} Y(f) Y^{*}(f), \\
& P_{x y}(f)=\frac{1}{L F_{s} U} X(f) Y^{*}(f),
\end{aligned}
$$

where $\mathrm{f}$ is frequency, $P_{x x}$ is the power spectral density of the input, $P_{y y}$ is the power spectral density of the output, $P_{x y}$ is the cross power spectrum of the input and output, $X$ is the Fourier transform of the windowed input signal, $Y$ is the Fourier transform of the windowed output signal, $L$ is the length of the corresponding signal, $F_{S}$ is the sampling frequency, $U$ is the energy of the Hanning window $\left(U=1 / L \sum w_{j}^{2}=0.375\right.$, where $\mathrm{w}_{j}$ is the $\mathrm{j}$ :th value of the Hanning window) and $*$ nominates complex conjugate. The time invariant transform function between input and output signals was calculated as follows:

$$
T F(f)=P_{x y}(f) / P_{x x}(f),
$$

which gives transfer function in a complex frequency space. In this study, the widely used Bode plot was used to illustrate the complex results, i.e., transfer functions' amplitude and phase parts are displayed separately in their own graphs and the amplitude part is shown in decibels. Furthermore, since this transfer function computation procedure is made separately for every 1-10 s segments of the longitudinal motion and the blood pressure data, the final presented transfer functions for individual subjects are the averages of the segmental transfer functions.

A magnitude squared coherence function was used to estimate how much of the output signal can be linearly explained by the input signal in different frequencies. The coherence function was computed as:

$$
C_{x y}(f)=\frac{\left|P_{x y}(f)\right|^{2}}{P_{x x}(f) P_{y y}(f)} .
$$

The coherence value over 0.5 was considered to be a significant sign of at least a partly linear relationship between input and output signals.

Amplitude and phase values on the heart rate frequency were measured from each subject's transfer function. This was done by defining a subject specific heartbeat band: the peak of the $P_{x x}$ of the blood pressure signal of each subject was detected and a $0.5 \mathrm{~Hz}( \pm 0.25 \mathrm{~Hz})$ wide band was centered on that peak. The average amplitude and phase values of the transfer function were computed from the heartbeat band, neglecting the values on frequencies where the magnitude squared coherence between input and output was lower than 0.5 .

\section{Stiffness Indices}

To define the stiffness of the artery, two different stiffness indices were measured: (1) Augmentation index adjusted for the heart rate of 75 beats per minute (Aix@75) was automatically computed from the aortic pressure waveform by the used SphygmoCor applanation tonometry system. (2) Young's elastic modulus $\left(\mathrm{E}_{Y}\right)$ was computed from the average diameter curve of the 5-min recording of the carotid artery as follows:

$$
E_{Y}=\frac{3\left(1+\frac{A_{\text {avg }}}{W C S A}\right) D_{\text {diastole }}^{2} \times P P_{\text {carotid }}}{D_{\text {systole }}^{2}-D_{\text {diastole }}^{2}}
$$

where $A_{\text {avg }}$ is the average lumen area, WCSA is the vessel wall cross-sectional area during diastole, computed from the intimamedia thickness by assuming cylindrical geometry. The intimamedia thickness measurement was done manually from the first video frame by drawing a line on top of the lumen-intima border and on top of media-adventitia border. $D_{\text {systole }}$ is the systolic diameter, $D_{\text {diastole }}$ is the diastolic diameter and $P P_{\text {carotid }}$ is the pulse pressure in carotid artery.

\section{Statistical Methods}

Some of the indices used in this study were not normally distributed and linearity was not expected between every index, thus Spearman's correlation analysis was used to find conformities between the measurements. A $p$-value lower than 0.05 was considered to be statistically significant.

\section{RESULTS}

The average amount of heart cycles per subject that were included from the 5-min recordings into the transfer function analysis was 260 , with the range being 132-322. The clinical characteristics and the blood pressures as well as the average heart rates are displayed in Table $\mathbf{1 .}$

The average power spectrums of the longitudinal motion of the intima-media complex and the adventitia layer as well as the blood pressure signal are displayed in Figure 3. The main power in all of the average spectrums is on the band $0-3 \mathrm{~Hz}$, with a large peak on the $1.1 \mathrm{~Hz}$ frequency, which is the frequency of the average heart rate within the population. In addition, a peak with low amplitude is visible above $2 \mathrm{~Hz}$ frequency in all power spectrums. In the power spectrums of the longitudinal motions, an additional smaller peak is visible on the frequency $0.2 \mathrm{~Hz}$.

The transfer function between the longitudinal motion of the intima-media complex and the adventitia layer is displayed in Figure 4. The amplitude part of the transfer function is negative in decibel scale throughout the observed spectrum $(0-5 \mathrm{~Hz})$, meaning that the longitudinal motion of the adventitia layer has lower amplitude than the intima-media complex. The maximum attenuation is on the frequency $1.0 \mathrm{~Hz}$, where the kinetic energy of the longitudinal motion of the adventitia layer drops $1.6 \mathrm{~dB}$ (31\%) compared to the intima-media complex, indicating that $17 \%$ of the longitudinal motion amplitude is lost in the transition on the frequency where the most power is in the longitudinal motion signals. The average standard deviation on the $0-5$ 

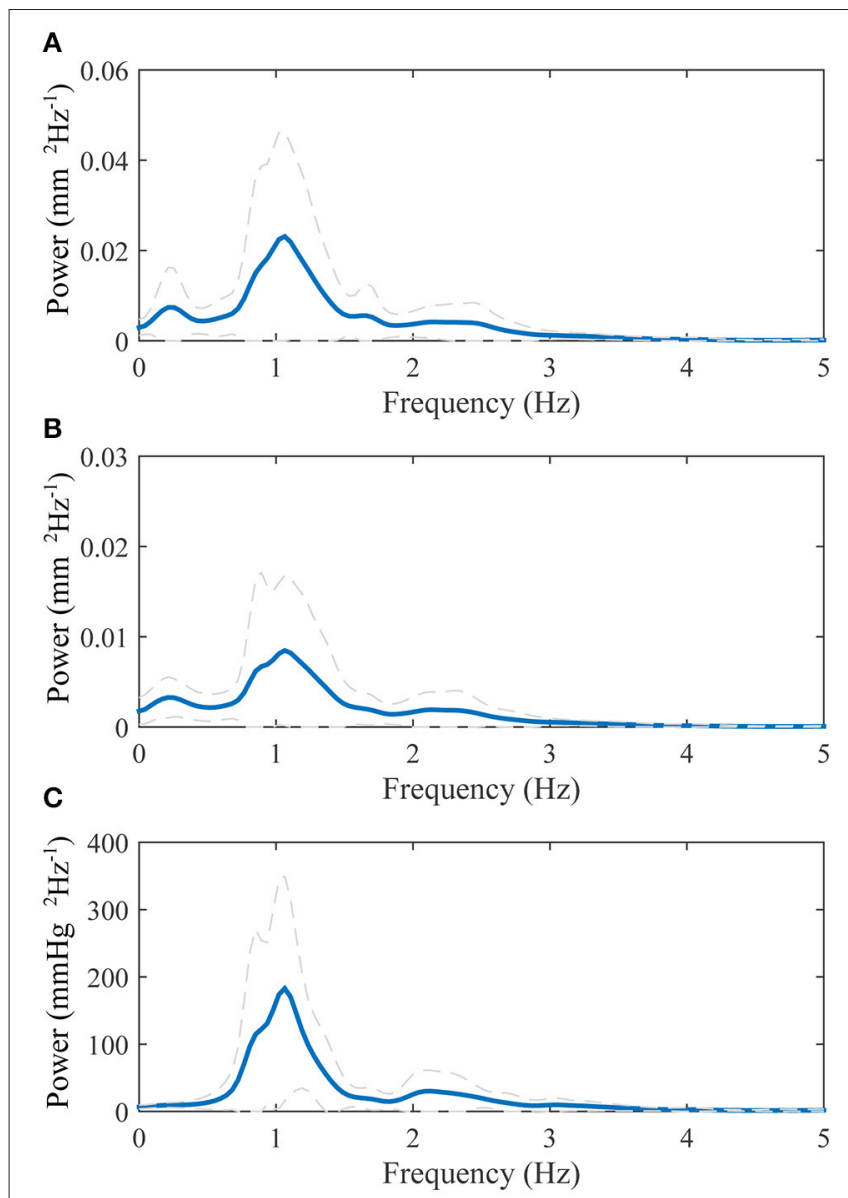

FIGURE 3 | Group averaged power spectrums: (A), longitudinal motion of the intima-media complex; (B), longitudinal motion of the adventitia layer; (C), blood pressure derived from the diameter change curve. The blue thick line displays the mean and the dashed gray lines displays the standard deviation.

$\mathrm{Hz}$ frequency of the amplitude part of the transfer function is approximately $1.4 \mathrm{~dB}$, indicating a large variation between individuals. The phase part of the transfer function is negative, indicating that the motion of the intima-media complex priors the adventitia layer. On the frequency $1.0 \mathrm{~Hz}$ the longitudinal motion of the intima-media complex priors the adventitia layer by 6.8 degrees $(18.9 \mathrm{~ms})$. The coherence of the transfer function is well above 0.5 throughout the observed spectrum.

The transfer function between the blood pressure and the longitudinal motion of the intima-media complex is displayed in Figure 5. The value of the amplitude part of the transfer function decreases in higher frequencies but there is also an additional, large notch in the amplitude part of the transfer function on the frequency $1.0 \mathrm{~Hz}$. The phase part of the transfer function is negative on the band from 0 to $3 \mathrm{~Hz}$. Although, on the band from 0.5 to $1.5 \mathrm{~Hz}$ the variation in the phase spectrum is large; the maximum standard deviation varies between -120 and 70 degrees, which may be due to large variation of the waveform shape of the longitudinal motion curve (antegrade and retrograde oriented movement). The coherence of the transfer function is

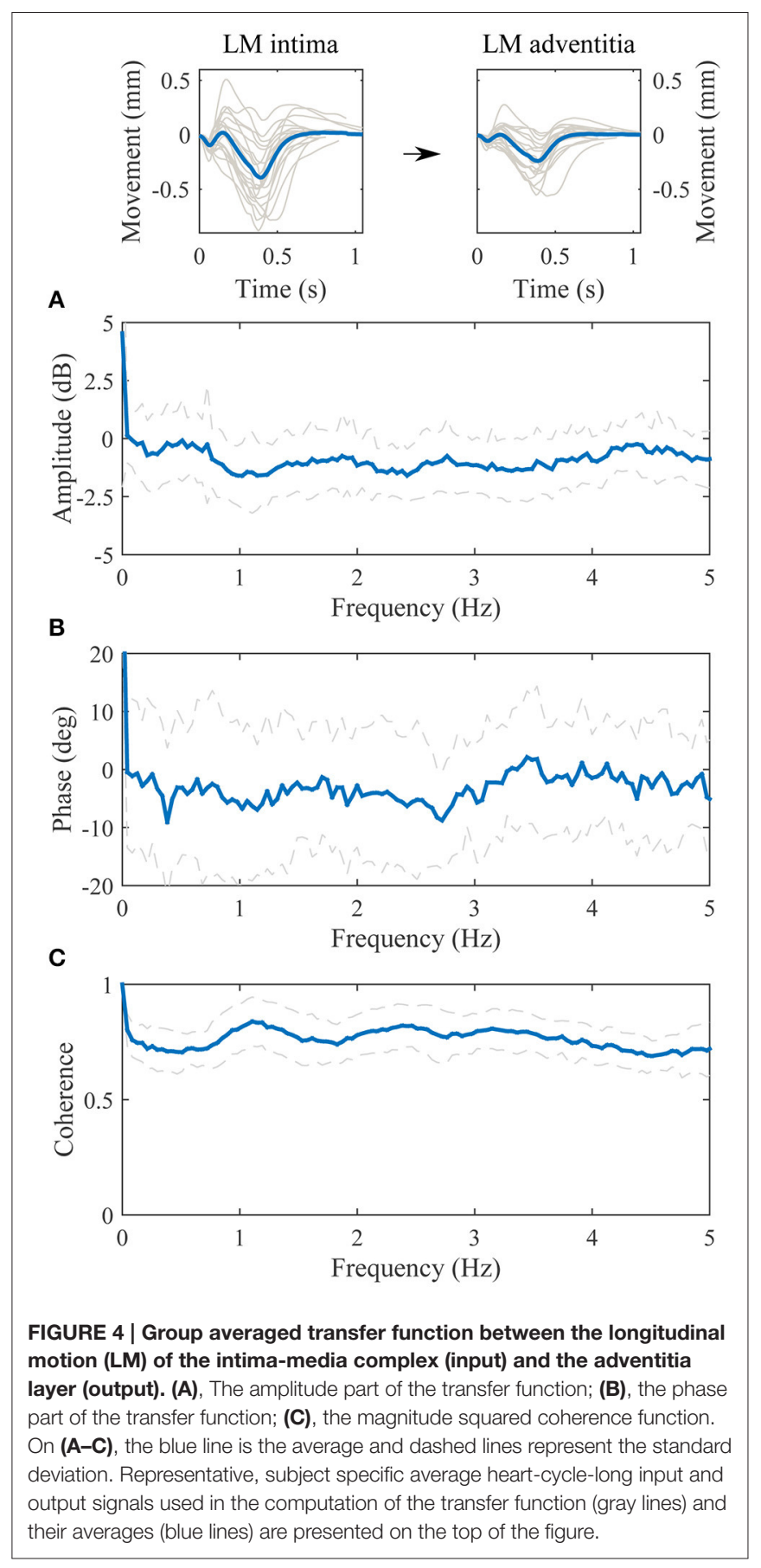

mainly above 0.5 throughout the spectrum, only merely under 0.5 on the independent frequency bands from 1.8 to $2.0 \mathrm{~Hz}$ and from 3.6 to $3.9 \mathrm{~Hz}$.

The population was divided into quartiles according to the main orientation of the longitudinal waveform $\left(\mathrm{IO}_{\mathrm{dev}}\right)$. Thirteen subjects out of 19 had a negative $\mathrm{IO}_{\mathrm{dev}}$ value i.e., the longitudinal motion was retrograde oriented. The transfer functions between the blood pressure and the longitudinal motion of the intimamedia complex, displaying the antegrade (upper quartile) and retrograde (lower quartile) oriented waveforms, respectively, 


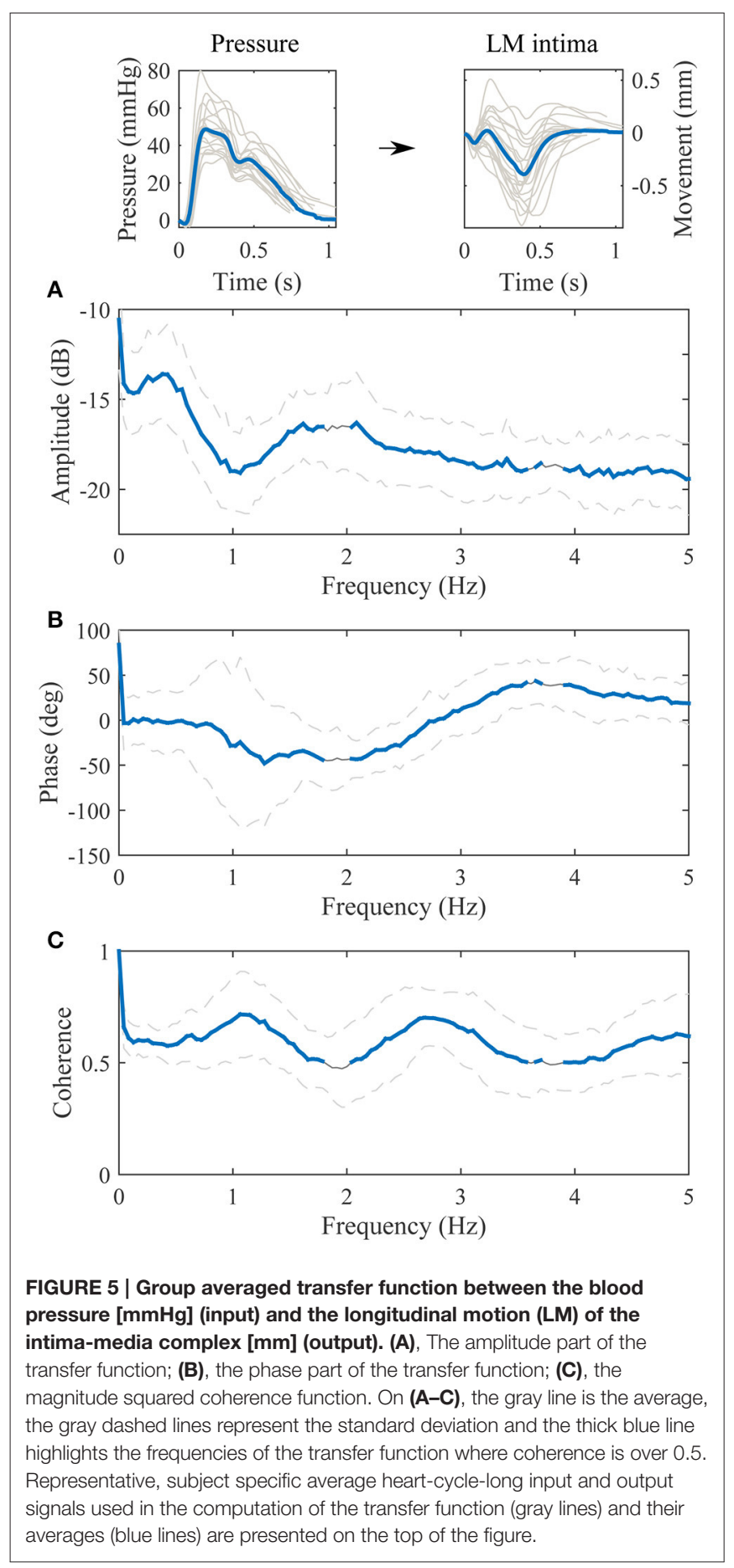

are presented in Figure 6. The amplitude parts of the quartile transfer functions are fairly similar to the corresponding whole population transfer function. The clearest difference between the transfer functions obtained from the antegrade and retrograde oriented waveforms is the phase part around $1 \mathrm{~Hz}$ : the antegrade transfer function has clear positive phase (peak at 37 degrees) and the retrograde transfer function has a clear negative phase (peak at -117 degrees). Another observable difference between the quartile transfer functions is the location of the maximum coherence: in the antegrade oriented population the maximum coherence is between 2 and $3 \mathrm{~Hz}$ whereas in the retrograde oriented population it is on $1 \mathrm{~Hz}$ and there is only a smaller peak on the $2-3 \mathrm{~Hz}$ band.

The correlations of the amplitude and the phase values on the individual heartbeat band and the arterial stiffness measures are presented in Table 2. The amplitude and the phase values of the transfer function between the longitudinal motion of the intimamedia complex and the adventitia layer did not display any correlation to known arterial stiffness indices. On the other hand, the phase part of the transfer function between the blood pressure and the longitudinal motion of the intima-media complex had a clear, indirect correlation with Aix@75 $(p<0.01)$ and $E_{Y}$ $(p<0.05)$.

There were no significant correlations between the referential arterial stiffness measures (Aix@75 and $\mathrm{E}_{Y}$ ) and the longitudinal motion parameters $\mathrm{IO}_{\mathrm{ampl}}(r=-0.013, p=0.957 ; r=-0.023, p$ $=0.926), \mathrm{IO}_{\text {ante }}(r=-0.023, p=0.926 ; r=-0.481, p=0.037)$, $\mathrm{IO}_{\text {retro }}(r=0.026, p=0.915 ; r=0.088, p=0.721)$, and $\mathrm{IO}_{\mathrm{dev}}(r$ $=-0.065, p=0.792 ; r=-0.319, p=0.183)$, respectively.

\section{DISCUSSION}

There are three primary findings in the present study. (1) In a healthy population, the longitudinal motion of the carotid artery wall occurs first in the intima-media complex and is then followed by the longitudinal motion of the adventitia layer. The delay between the motions is 6.8 degrees $(\sim 18.9 \mathrm{~ms})$ and the attenuation is $1.6 \mathrm{~dB}$ in the heartbeat frequency $(1.0 \mathrm{~Hz})$. (2) The blood pressure (or the diameter change) in the common carotid artery has a partly linear relationship to the longitudinal motion of the intima-media complex and is thus a potential initiating force for the longitudinal motion. (3) The phase difference between the blood pressure signal and the longitudinal motion of the intima-media complex on the heartbeat band has a potential relationship to arterial stiffness.

The power spectrums of the longitudinal motion and the blood pressure signal both display a peak on the frequency $1.1 \mathrm{~Hz}$, which also is the measured average heart rate frequency within the study population. Since the work of the heart muscle is the origin for the blood pressure and blood flow in arteries, it is logical that most of the power within the longitudinal motion of the common carotid artery wall is on the same frequency band that the heart muscle operates. In addition, a lower peak is visible in the power spectrums above the $2 \mathrm{~Hz}$ frequency. This reflects the multiphase nature of the blood pressure and longitudinal motion signals during a cardiac cycle, such as the change of direction of the longitudinal motion during systole. In the power spectrums of the longitudinal motion of the intimamedia complex and the adventitia layer, there is also a smaller peak visible on the $0.2 \mathrm{~Hz}$ frequency. The effect of breathing to the longitudinal wall motion has been demonstrated previously (Cinthio et al., 2005). The peak in the power spectrums on the frequency $0.2 \mathrm{~Hz}$, presented here, is on the band $0.2-0.33$ $\mathrm{Hz}$ where the free breathing occurs (Ball et al., 2014) and thus 


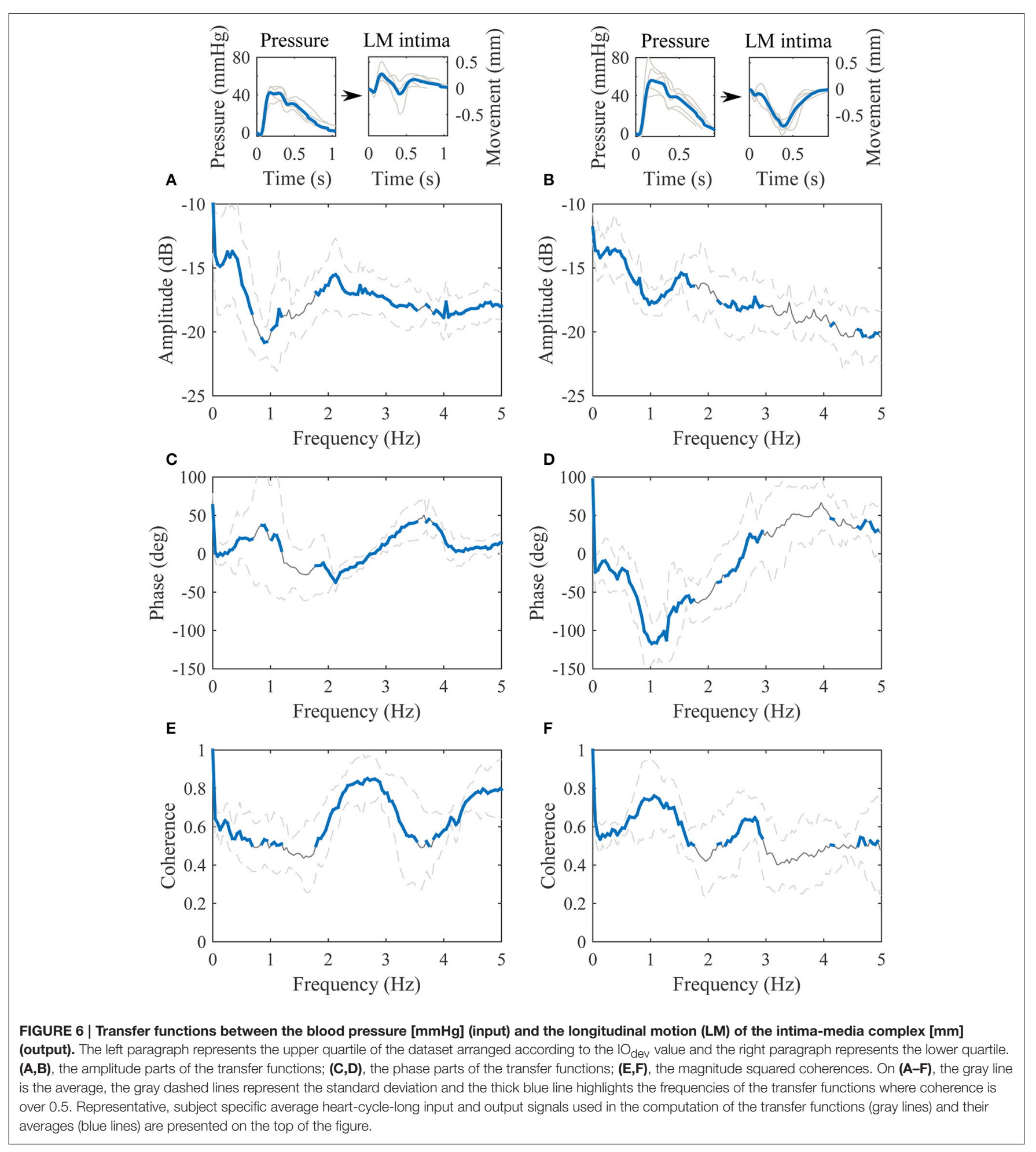

is possibly confirming observation that breathing modifies the longitudinal motion of the common carotid artery wall, although breathing was not directly measured in this study.

Our results show that the longitudinal motion of the common carotid artery wall is first occurring in the intima-media complex and is then followed by the longitudinal motion of the adventitia layer. The average delay between the longitudinal motions is 6.8 degrees on the $1.0 \mathrm{~Hz}$ frequency, which translates into $18.9 \mathrm{~ms}$. The media layer is connected to the adventitia by an external elastic lamina which is composed of condensed sheets of elastic 
TABLE 2 | Correlations between the transfer function parameters defined from the heartbeat band and arterial stiffness indices as well as longitudinal amplitude parameters.

\begin{tabular}{|c|c|c|c|c|c|c|}
\hline & Aix@75 & $E_{Y}$ & $\mathrm{IO}_{\mathrm{ampl}}$ & $1 O_{\text {ante }}$ & $1 \mathrm{O}_{\text {retro }}$ & $10_{\text {dev }}$ \\
\hline \multicolumn{7}{|c|}{ TF: INTIMA-MEDIA -> ADVENTITIA } \\
\hline Amplitude of the heartbeat band & 0.245 & 0.139 & -0.411 & -0.072 & -0.270 & 0.209 \\
\hline Phase of the heartbeat band & 0.048 & 0.221 & 0.214 & -0.221 & 0.226 & -0.144 \\
\hline \multicolumn{7}{|c|}{ TF: BLOOD PRESSURE -> INTIMA-MEDIA } \\
\hline Amplitude of the heartbeat band & -0.033 & -0.160 & $0.833^{\star \star \star}$ & $-0.490^{\star}$ & $0.886^{\star * *}$ & $-0.730^{* * *}$ \\
\hline Phase of the heartbeat band & $-0.658^{\star \star}$ & $-0.495^{\star}$ & -0.379 & 0.432 & -0.443 & $0.560^{\star}$ \\
\hline
\end{tabular}

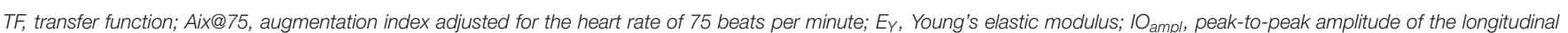

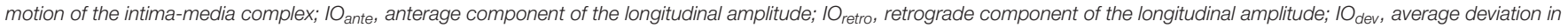
longitudinal direction during a heart cycle. ${ }^{*} p<0.05,{ }^{* *} p<0.01$, ${ }^{* * *} p<0.001$.

fibers (Stevens and Lowe, 1997), thus it is possible that the elastic fibers drag the adventitia layer along, as the intima-media complex moves in the longitudinal direction. According to the amplitude part of the transfer function, 31\% $(1.6 \mathrm{~dB})$ of the longitudinal kinetic energy of the intima-media complex is lost to drag the adventitia layer along. Since the power (energy) of the longitudinal motion signal is related to the square of the longitudinal amplitude, the $31 \%$ lost in power equals to $17 \%$ lost in longitudinal motion amplitude. The abatement of the longitudinal motion occurs throughout the observed spectrum $(0-5 \mathrm{~Hz})$ and it reaches its maximum on the $1.0 \mathrm{~Hz}$ frequency. The standard deviation in the amplitude part of the transfer function between the longitudinal motion of the intima-media complex and the adventitia layer is on average $1.4 \mathrm{~dB}$ between frequencies $0 \mathrm{~Hz}$ and $5 \mathrm{~Hz}$, meaning that there is quite large variation in the abatement of the longitudinal wall motion between individuals. Since the amplitude of the longitudinal carotid wall motions have been previously connected to arterial stiffness (Zahnd et al., 2011a; Taivainen et al., 2015; Yli-Ollila et al., 2015), this deviation observed in the transfer functions could be related to arterial stiffness but according to our results with 19 healthy individuals, such connection to known arterial stiffness parameters was not found. The possible connection to arterial stiffness should be studied with a larger population, including unhealthy subjects.

The diameter change of the common carotid artery behaves similarly as the blood pressure signal within the artery (Barnett et al., 1961; Patel et al., 1963; Sugawara et al., 2000; Giannattasio et al., 2008) and thus the diameter change was used here as a base to model the blood pressure and to define the transfer function between the blood pressure and the longitudinal motion of the intima-media complex. The coherence between the blood pressure signal and the longitudinal wall motion of the carotid is mainly above 0.5 . This is a sign of a partly linear relationship between the signals. According to our results, the amplitude part of the transfer function decreases with higher frequencies and with an additional notch on the $1.0 \mathrm{~Hz}$ frequency. This decreasing amplitude within higher frequencies and the notch on the $1 \mathrm{~Hz}$ frequency is observed for both antegrade and retrograde oriented wall motions. The phase of the transfer function on the band from 0.5 to $1.5 \mathrm{~Hz}$ has large variation within the study population and it displays a clear correlation to previously known arterial stiffness parameters. The correlation between the $\mathrm{IO}_{\mathrm{dev}}$ and the phase value of the transfer function in the heartbeat band is 0.560 . This is due to the large variance in the main direction of the longitudinal motion among the individuals. A positive $1 \mathrm{~Hz}$ blood pressure signal and a simultaneous negative (retrograde oriented) $1 \mathrm{~Hz}$ longitudinal motion causes approximately 180 degree phase shift into the transfer function and if the longitudinal motion is positive (antegrade oriented) the phase shift is closer to 0 degrees. The phase difference on the frequency band can also be seen in the transfer functions of the lower and upper quartile of the population, arranged according to $\mathrm{IO}_{\mathrm{dev}}$ value. The antegrade oriented quartile has a positive phase and the retrograde oriented quartile has a negative phase on the band. Nevertheless, the main direction does not explain the phase in the heartbeat band completely but some additional phase difference is also occurring before the longitudinal motion achieves its absolute peak value. This additional phase difference seems to be related to the early arterial stiffening because the phase values in the heartbeat band of the transfer function display a clear correlation to the known stiffness indices but the $\mathrm{IO}_{\mathrm{dev}}$ does not. This is in line with our previous finding that the shape of the waveform of the longitudinal motion is associated with early stiffening in the common carotid artery (Yli-Ollila et al., 2016).

The higher coherence on the frequencies between 2 and $3 \mathrm{~Hz}$ observed in the transfer function of the upper quartile of the data arranged according to the $\mathrm{IO}_{\mathrm{dev}}$ value is a possible indirect sign of a biphasic longitudinal motion. The coherence on the lower quartile transfer function was significantly lower compared to the upper quartile transfer function on the $2-3 \mathrm{~Hz}$ band. The difference can also be seen in Figure 6 where the waveforms with a positive $\mathrm{IO}_{\mathrm{dev}}$ value oscillate more backwards and forwards than the waveforms with a negative $\mathrm{IO}_{\mathrm{dev}}$ value. The difference in the coherence is clear but its connection to the biphasic longitudinal motion requires further studies. What is common for all the longitudinal motion waveforms is that they all have a tendency to steer toward the antegrade direction when the blood pressure reaches its maximum, although the amplitude of the antegrade peak in the motion waveform varies a lot. Another similarity with all the waveforms is the steer toward the retrograde direction roughly when the diastolic phase of the heart cycle begins. Similar observations have been made elsewhere (Cinthio et al., 2006; Au et al., 2016). 
When interpreting our results one must remember that this is mainly a methodological study presenting a new method to be used for studying longitudinal wall kinetics and cardiovascular diseases, but for clinical use the method needs to be validated with a large and carefully characterized study population including unhealthy subjects. Another notable fact is that the transfer function only describes the linear timeinvariant relationship between the measured input and output, i.e., between the longitudinal motion of the intima-media complex and the adventitia layer or between the blood pressure and the longitudinal motion of the intima-media complex. This means that there can possibly be an additional nonlinear relationship between the signals that cannot be observed here. In biological systems, nonlinear input-output characteristics are always present but the coherence functions presented here; serve as evidence that the relationships between the longitudinal motion of the intima-media complex and adventitia layer and between the blood pressure and the longitudinal motion of the intima-media complex are mainly linear. In addition to the nonlinearity, there are other reasons for the lower coherence, such as noise in the measurement signal, especially in higher frequencies. However, in this study the power of the frequencies higher than $5 \mathrm{~Hz}$ was negligible. The output can also be due to multiple inputs, which is the most likely reason for the lower coherence in the transfer function between the blood pressure and the longitudinal motion of the intima-media.

\section{CONCLUSION}

This study describes for the first time how the energy from the blood pressure transfers into the longitudinal motion of the innermost carotid wall layer and from there into the longitudinal motion of the outermost wall layer. Also, the early arterial stiffening seems to modify the phase difference between the carotid blood pressure and the longitudinal motion of the common carotid artery wall. The results give new insights for

\section{REFERENCES}

Ahlgren, A. R., Cinthio, M., Persson, H. W., and Lindström, K. (2012). Different patterns of longitudinal displacement of the common carotid artery wall in healthy humans are stable over a four-month period. Ultrasound Med. Biol. 38, 916-925. doi: 10.1016/j.ultrasmedbio.2012.02.005

Albinsson, J., Brorsson, S., Ahlgren, A. R., and Cinthio, M. (2014). Improved tracking performance of lagrangian block-matching methodologies using block expansion in the time domain: in silico, phantom and in vivo evaluations. Ultrasound Med. Biol. 40, 2508-2520. doi: 10.1016/j.ultrasmedbio.2014.05.010

Au, J. S., Ditor, D. S., MacDonald, M. J., and Stöhr, E. J. (2016). Carotid artery longitudinal wall motion is associated with local blood velocity and left ventricular rotational, but not longitudinal, mechanics. Physiol. Rep. 4:e12872. doi: $10.14814 /$ phy2.12872

Ball, J. W., Dains, J. E., Flynn, J. A., Solomon, B. S., and Stewart, R. W. (2014). Seidel's Guide to Physical Examination. St. Louis, MO: Elsevier Health Sciences.

Barnett, G. O., Mallos, A. J., and Shapiro, A. (1961). Relationship of aortic pressure and diameter in the dog. J. Appl. Physiol. 16, 545-548.

Cinthio, M., Ahlgren, A. R., Bergkvist, J., Jansson, T., Persson, H. W., and Lindström, K. (2006). Longitudinal movements and resulting shear strain of the arterial wall. Am. J. Physiol. Heart Circ. Physiol. 291, H394-H402. doi: 10.1152/ajpheart.00988.2005 deriving the driving force of the longitudinal wall motion, for better understanding of the longitudinal wall kinetics and for developing novel methods to detect the early signs of arterial stiffening. Our study population was small and healthy and thus a larger clinical validation is needed to confirm the tentative findings and to study how the transfer functions are modified by different cardiovascular diseases.

\section{ETHICS STATEMENT}

Fully informed written consent was obtained from each participant and the Ethics Committee of the University of Eastern Finland and Kuopio University Hospital approved the used study protocol.

\section{AUTHOR CONTRIBUTIONS}

HY, MT, TPL, and TML designed the experiment and HY, TPL, and TML acquired the data. HY, MT, TPL, and TML contributed to the analysis and interpretation of data. HY, MT, TPL, and TML participated in the elaboration of the manuscript and gave the final approval for submission and publication, being accountable for all aspects of the present work.

\section{FUNDING}

We acknowledge the financial support of the Kuopio University Hospital (EVO 5031320, 5031316 and VTR 5031356) and the University of Eastern Finland. In addition, we would like to express our gratitude toward the following foundations for financial support: Science Foundation of Kuopio University Hospital, Aarne and Aili Turunen Foundation, Foundation for the Promotion of Technological Advances, Aleksanteri Mikkonen Foundation, Finnish Foundation for Cardiovascular Research and Antti and Tyyne Soininen Foundation.

Cinthio, M., Ahlgren, A. R., Jansson, T., Eriksson, A., Persson, H. W., and Lindström, K. (2005). Evaluation of an ultrasonic echo-tracking method for measurements of arterial wall movements in two dimensions. IEEE Trans. Ultrason. Ferroelectr. Freq. Control 52, 1300-1311. doi: 10.1109/TUFFC.2005.1509788

Giannattasio, C., Salvi, P., Valbusa, F., Kearney-Schwartz, A., Capra, A., Amigoni, M., et al. (2008). Simultaneous measurement of beat-to-beat carotid diameter and pressure changes to assess arterial mechanical properties. Hypertension 52, 896-902. doi: 10.1161/HYPERTENSIONAHA.108.116509

Golemati, S., Sassano, A., Lever, M. J., Bharath, A. A., Dhanjil, S., and Nicolaides, A. N. (2003). Carotid artery wall motion estimated from B-mode ultrasound using region tracking and block matching. Ultrasound Med. Biol. 29, 387-399. doi: 10.1016/S0301-5629(02)00760-3

Hu, H. H., Kuo, T. B., Wong, W. J., Luk, Y. O., Chern, C. M., Hsu, L. C., et al. (1999). Transfer function analysis of cerebral hemodynamics in patients with carotid stenosis. J. Cereb. Blood Flow Metab. 19, 460-465. doi: 10.1097/00004647-199904000-00012

Karamanoglu, M., and Feneley, M. P. (1996). Derivation of the ascending aorticcarotid pressure transfer function with an arterial model. Am. J. Physiol. 271, H2399-H2404.

Kuo, T. B., Chern, C. M., Sheng, W. Y., Wong, W. J., and Hu, H. H. (1998). Frequency domain analysis of cerebral blood flow velocity and its correlation 
with arterial blood pressure. J. Cereb. Blood Flow Metab. 18, 311-318. doi: 10.1097/00004647-199803000-00010

Lipsitz, L. A., Mukai, S., Hamner, J., Gagnon, M., and Babikian, V. (2000). Dynamic regulation of middle cerebral artery blood flow velocity in aging and hypertension. Stroke 31, 1897-1903. doi: 10.1161/01.STR.31. 8.1897

Nilsson, T., Ahlgren, A., Jansson, T., Persson, H. W., Nilsson, J., Lindstrom, K., et al. (2010). "A method to measure shear strain with high spatial resolution in the arterial wall non-invasively in vivo by tracking zero-crossings of B-mode intensity gradients," in Proceedings IEEE Ultrason Symposium (San Diego, CA: IEEE), 491-494.

Patel, D. J., De Freitas, F. M., Greenfield, J. C. Jr., and Fry, D. L. (1963). Relationship of radius to pressure along the aorta in living dogs. J. Appl. Physiol. 18, $1111-1117$.

Persson, M., Ahlgren, A. R., Jansson, T., Eriksson, A., Persson, H. W., and Lindström, K. (2003). A new non-invasive ultrasonic method for simultaneous measurements of longitudinal and radial arterial wall movements: first in vivo trial. Clin. Phys. Funct. Imaging 23, 247-251. doi: 10.1046/j.1475-097X.2003.00504.x

Segers, P., Carlier, S., Pasquet, A., Rabben, S. I., Hellevik, L. R., Remme, E., et al. (2000). Individualizing the aorto-radial pressure transfer function: feasibility of a model-based approach. Am. J. Physiol. Heart Circ. Physiol. 279, H542-H549. Available online at: http://ajpheart.physiology.org/content/279/2/H542

Stevens, A., and Lowe, J. S. (1997). Human Histology. London: Mosby.

Sugawara, M., Niki, K., Furuhata, H., Ohnishi, S., and Suzuki, S. (2000). Relationship between the pressure and diameter of the carotid artery in humans. Heart Vessels 15, 49-51. doi: 10.1007/PL00007261

Svedlund, S., Eklund, C., Robertsson, P., Lomsky, M., and Gan, L. M. (2011). Carotid artery longitudinal displacement predicts 1-year cardiovascular outcome in patients with suspected coronary artery disease. Arterioscler. Thromb. Vasc. Biol. 31, 1668-1674. doi: 10.1161/ATVBAHA.111.222901

Svedlund, S., and Gan, L. (2011a). Longitudinal wall motion of the common carotid artery can be assessed by velocity vector imaging. Clin. Physiol. Funct. Imaging 31, 32-38. doi: 10.1111/j.1475-097X.2010.00976.x

Svedlund, S., and Gan, L. (2011b). Longitudinal common carotid artery wall motion is associated with plaque burden in man and mouse. Atherosclerosis 217, 120-124. doi: 10.1016/j.atherosclerosis.2011.02.046

Taivainen, S. H., Yli-Ollila, H., Juonala, M., Kähönen, M., Raitakari, O. T., Laitinen, T. M., et al. (2015). Interrelationships between indices of longitudinal movement of the common carotid artery wall and the conventional measures of subclinical arteriosclerosis. Clin. Physiol. Funct. Imaging. doi: 10.1111/cpf.12305. [Epub ahead of print].
Tat, J., Au, J. S., Keir, P. J., and MacDonald, M. J. (2015). Reduced common carotid artery longitudinal wall motion and intramural shear strain in individuals with elevated cardiovascular disease risk using speckle tracking. Clin. Physiol. Funct. Imaging. doi: 10.1111/cpf.12270. [Epub ahead of print].

Yli-Ollila, H., Laitinen, T., Weckström, M., and Laitinen, T. (2013). Axial and radial waveforms in common carotid artery: an advanced method for studying arterial elastic properties in ultrasound imaging. Ultrasound Med. Biol. 39, 1168-1177. doi: 10.1016/j.ultrasmedbio.2013.01.018

Yli-Ollila, H., Laitinen, T., Weckström, M., and Laitinen, T. M. (2015). New indices of arterial stiffness measured from longitudinal motion of common carotid artery in relation to reference methods, a pilot study. Clin. Physiol. Funct. Imaging 36, 376-388. doi: 10.1111/cpf.12240

Yli-Ollila, H., Tarvainen, M. P., Laitinen, T. P., and Laitinen, T. M. (2016). Principal component analysis of the longitudinal carotid wall motion in association with vascular stiffness: a pilot study. Ultrasound Med. Biol. 42, 2873-2886. doi: 10.1016/j.ultrasmedbio.2016.07.020

Zahnd, G., Boussel, L., Marion, A., Durand, M., Moulin, P., Sérusclat, A., et al. (2011a). Measurement of two-dimensional movement parameters of the carotid artery wall for early detection of arteriosclerosis: a preliminary clinical study. Ultrasound Med. Biol. 37, 1421-1429. doi: 10.1016/j.ultrasmedbio.2011.05.843

Zahnd, G., Orkisz, M., Serusclat, A., and Vray, D. (2011b). "Minimal-path contours combined with speckle tracking to estimate $2 \mathrm{D}$ displacements of the carotid artery wall in B-mode imaging," in Ultrasonics Symposium (IUS), 2011 IEEE International (Orlando, FL: IEEE), 732-735.

Zahnd, G., Vray, D., Sérusclat, A., Alibay, D., Bartold, M., Brown, A., et al. (2012). Longitudinal displacement of the carotid wall and cardiovascular risk factors: associations with aging, adiposity, blood pressure and periodontal disease independent of cross-sectional distensibility and intima-media thickness. Ultrasound Med. Biol. 38, 1705-1715. doi: 10.1016/j.ultrasmedbio.2012.05.004

Conflict of Interest Statement: The authors declare that the research was conducted in the absence of any commercial or financial relationships that could be construed as a potential conflict of interest.

Copyright (๑ 2016 Yli-Ollila, Tarvainen, Laitinen and Laitinen. This is an openaccess article distributed under the terms of the Creative Commons Attribution License (CC BY). The use, distribution or reproduction in other forums is permitted, provided the original author(s) or licensor are credited and that the original publication in this journal is cited, in accordance with accepted academic practice. No use, distribution or reproduction is permitted which does not comply with these terms. 